\title{
Simultaneous measurement of the average ion-induced electron emission yield and the mean charge for isotachic ions in carbon foils
}

\author{
A. M. Arrale \\ Eaton Corporation, Semiconductor Equipment Division, 2433 Rutland Drive, Austin, Texas 78758-5285 \\ Z. Y. Zhao, J. F. Kirchhoff, D. L. Weathers, F. D. McDaniel, and S. Matteson \\ Ion Beam Modification and Analysis Laboratory, Department of Physics and Center for Materials Characterization, \\ University of North Texas, Denton, Texas 76203
}

(Received 27 January 1995)

\begin{abstract}
Knowledge of the incident ion's atomic number $\left(Z_{1}\right)$ dependence of ion-induced electron emission yields can be the basis for a general understanding of ion-atom interaction phenomena and, in particular, for the design of $Z_{1}$-sensitive detectors that could be useful, for example, in the separation of isobars in accelerator mass spectrometry. The $Z_{1}$ dependence of ion-induced electron emission yields, $\gamma$, has been investigated using heavy ions $\mathrm{C}^{3+}, \mathrm{O}^{3+}, \mathrm{F}^{+3}, \mathrm{Na}^{3+}, \mathrm{Al}^{3+}, \mathrm{Si}^{3+}, \mathrm{P}^{3+}, \mathrm{S}^{3+}, \mathrm{Cl}^{3+}, \mathrm{K}^{3+}, \mathrm{Ti}^{3+}, \mathrm{Cr}^{3+}, \mathrm{Mn}^{4+}, \mathrm{Fe}^{4+}$, $\mathrm{Co}^{4+}, \mathrm{Ni}^{4+}, \mathrm{Cu}^{4+}, \mathrm{Ga}^{4+}, \mathrm{As}^{5+}, \mathrm{Br}^{5+}, \mathrm{Ru}^{7+}, \mathrm{Ag}^{7+}, \mathrm{Sn}^{7+}$, and $\mathrm{I}^{8+}$ of identical velocity $\left(v=2 v_{0}\right.$, where $v_{0}$ is the Bohr velocity) normally incident on $50 \mu \mathrm{g} / \mathrm{cm}^{2}$ sputter-cleaned carbon foils. Measured yields as a function of $Z_{1}$ reveal an oscillatory behavior with pronounced maxima and minima. Contrary to previously reported yields that assumed a monotonically increasing empirical mean charge state for the exiting ion, the present work indicates the $Z_{1}$ oscillations in the experimentally measured yields, a fact masked in previous work. The strong $Z_{1}$ oscillations can only be observed by simultaneous measurement of the yield and the mean charge state.

PACS number(s): 34.50.Dy, 79.20.-m
\end{abstract}

\section{INTRODUCTION}

It is a well-known phenomenon that electrons are emitted when energetic ions impinge on solid targets [1-4]. Not only is the understanding of the processes that lead to the emission of electrons of fundamental interest, but also the emission has a wide range of applications in such areas as timeof-flight spectroscopy, atomic number identification, plasma wall effects in nuclear fusion, electron microscopy, Auger spectroscopy, semiconductor physics, and in photoelectric and electron multiplier devices. Because of the dependence of the ion-induced electron emission (IIEE) yield on the type of incident ion, it has been suggested [5] that electrons emitted from target foils can be used for atomic number $\left(Z_{1}\right)$ identification of ions. In accelerator mass spectroscopy (AMS), where trace elements in electronic materials may be characterized [6], pursuing ion-induced electron emission methods could further help to identify elements with the same mass number but different atomic numbers (isobars). In some particle irradiation experiments, if not properly accounted for, emitted electrons complicate the absolute beam current measurement $[7,8]$.

The IIEE yield $\gamma$, defined as the total number of electrons emitted per incident ion, can proceed by two different mechanisms: potential-energy emission (PE) and kineticenergy emission (KEE). For ion velocities above $10^{7} \mathrm{~cm} / \mathrm{s}$, KEE is the dominant cause of the yield $[9,10]$ as opposed to PE $[9,11,12]$ which is beyond the scope of the present work. In the KEE process, it is the kinetic energy transferred to the target electrons that causes the production, transport, and the emission of electrons from the target material $[3,4]$.

It has long been an acknowledged fact that IIEE depends on stopping powers, and this assumption is best evidenced by the semiempirical calculations of Sternglass [3]. Even the more recent calculations done by Schou $[4,13]$ and Sigmund and Tougaard [14] show the yield to be proportional to the stopping power. For the case of protons over a wide range of projectile energies, the proportionality between the yield and stopping power has been confirmed [15-17]. For heavier ions, others have shown [18-20] that in certain cases, the yield and stopping powers have the same velocity dependence. However, for a given energy, their yields do not show the same $Z_{1}$ dependence.

Clerc et al. [5] proposed over 20 years ago that ioninduced electrons can be used for $Z_{1}$ detection; however, no further attention has been directed to this area to determine if such detection is feasible. One way to test the above proposal is to establish if the electron emission yield is sensitive to the type of ion by using a wide range of projectile ions. If a meaningful difference is portrayed in the electron emission yield of, for example, ions differing in atomic number by at least 1 , then an ion-induced electron emission technique could be a useful tool in identifying isobars.

The $Z_{1}$ dependence of the electron yield from bulk metallic targets induced by low-energy $(<50 \mathrm{keV})$ ions has been published in the past by other authors [21-23]. They have reported a periodic variation of the yield that is not correlated with the stopping power of the target. They found that the type of target material affects only the amplitude of the oscillation and not the location of the maximum. These studies were performed at extremely low ion velocities $\left(v<v_{0}\right.$, where $v_{0}=2.19 \times 10^{8} \mathrm{~cm} / \mathrm{s}$ is the Bohr velocity, the classical velocity of the electron in the ground state of hydrogen). The present work reports investigations at a much higher velocity.

The energy loss of the ion is a function of its charge state. 
Therefore, whenever appropriate, we have examined whether the initial charge state of the ion has any effect on the yield - at least in the energy range in which we are working. Earlier results from other authors $[15,19]$ seem to suggest some dependence of the yield on the charge state. We shall clearly establish the relevance and magnitude of this effect.

On the following pages, we present the often referenced theories and the experimental techniques pertaining to the field of ion-induced kinetic electron emission. The experimental procedures for the simultaneous measurement of accurate and reproducible yields, and the mean charge state are discussed in Sec. II. In Sec. III, the forward, backward, and total IIEE yields induced by swift $\left(v=2 v_{0}\right)$ heavy ions $\left(\mathrm{C}^{3+}, \mathrm{O}^{3+}, \mathrm{F}^{3+}, \mathrm{Na}^{3+}, \mathrm{Al}^{3+}, \mathrm{Si}^{3+}, \mathrm{P}^{3+}, \mathrm{S}^{3+}, \mathrm{Cl}^{3+}\right.$, $\mathrm{K}^{3+}, \mathrm{Ti}^{3+}, \mathrm{Cr}^{3+}, \mathrm{Mn}^{4+}, \mathrm{Fe}^{4+}, \mathrm{Co}^{4+}, \mathrm{Ni}^{4+}, \mathrm{Cu}^{4+}$, $\mathrm{Ga}^{4+}, \mathrm{As}^{5+}, \mathrm{Br}^{5+}, \mathrm{Ru}^{7+}, \mathrm{Ag}^{7+}, \mathrm{Sn}^{7+}$, and $\mathrm{I}^{8+}$ ) incident on sputter-cleaned carbon foils are presented and discussed in the framework of existing theories.

\section{EXPERIMENT}

Present experiments were done using the National Electrostatics Corporation 9SDH $3 \mathrm{MV}$ tandem accelerator in the Ion Beam Modification and Analysis Laboratory at the University of North Texas in Denton, Texas. The IIEE chamber has an ultimate pressure below $10^{-9}$ Torr maintained by two differential pumping sections, evacuated by turbomolecular pumps. The details of the experimental apparatus for measurement of the IIEE yield are given in Ref. [24]. The apparatus consists of forward and backward suppressors biased to $-2500 \mathrm{~V}$, forward and backward collectors biased to +100 $\mathrm{V}$, and a Faraday cup, all insulated from one another and from the ground.

The total IIEE yield $\left(\gamma_{T}\right)$ can be experimentally computed by equating the net charge at the target [2] to the sum of the incoming and outgoing charge. The backward, forward, and the total yields are given as follows [24]:

$$
\begin{gathered}
\gamma_{b}=\left(\frac{Q_{b}}{Q_{\mathrm{FC}}}\right) \bar{q}_{f}, \quad \gamma_{f}=\left(\frac{Q_{f}}{Q_{\mathrm{FC}}}\right) \overline{q_{f}}, \\
\gamma_{T}=\left(\frac{Q_{t}}{Q_{\mathrm{FC}}}\right) \bar{q}_{f}+\left(\bar{q}_{f}-q_{i}\right) \quad \text { also } \gamma_{T}=\gamma_{b}+\gamma_{f},
\end{gathered}
$$

where $q_{i}$ is the incoming charge state of the ion, and $\bar{q}_{f}$ is the mean charge state of the ion after exiting the foil. $Q_{t}, Q_{b}$, $Q_{f}$, and $Q_{\mathrm{FC}}$ are the charge collected from the target, backward collector, forward collector, and the Faraday cup, respectively, for a large number of ions. The total yield can be determined by two independent means as seen in Eq. (2). In this way, a check of the internal consistency of the results can be made. The difference was typically less than $0.3 \%$ for our measurements. The mean charge state could be determined from our experimental result using the following relation:

$$
\overline{q_{f}}=\left(\frac{Q_{\mathrm{FC}}}{Q_{\mathrm{ion}}}\right) q_{i} .
$$

Note that $Q_{\mathrm{FC}}$ and $Q_{\text {ion }}$ are simultaneously measured while the foil is in place. The charge of the ion $\left(Q_{\text {ion }}\right)$ is then the algebraic sum of all charges given by

$$
Q_{\text {ion }}=\left|Q_{\mathrm{FC}}\right|+\left|Q_{t}\right|-\left|Q_{b}\right|-\left|Q_{f}\right|
$$

Yield measurements obtained at a pressure above $10^{-9}$ Torr vacuum conditions are known to be higher than yields obtained under ultrahigh vacuum conditions. In order to measure a consistent and reproducible yield we have studied the yields from contaminated as well as in situ sputtercleaned surfaces, using argon gas as the sputtering agent [24]. The yield decreased as the target was bombarded with the ion and saturated to a constant low value, which is a characteristic of a clean surface. The dependence of the yield on vacuum and beam conditions has been reported in detail previously [24]. In the present work, the conditions were adjusted to assure reproducible and accurate results representing the "clean" carbon surface.

\section{RESULTS AND DISCUSSION}

In order to measure the $Z_{1}$ dependence of the IIEE yields, the present work utilized a wide range of ions of identical velocity $\left(v=2 v_{0}\right)$ incident normally on a $50 \mu \mathrm{g} / \mathrm{cm}^{2}$ sputter-cleaned carbon foil. By yield the authors mean the saturated yield obtained when the ion beam is allowed to sputter the foil for 3 to $4 \mathrm{~h}$. The saturated yield $\left(\gamma_{s}\right)$ has already been shown to be equal to the clean yield $\left(\gamma_{c}\right)$ under the experimental conditions of this work [24]. The forward $\left(\gamma_{f}\right)$, backward $\left(\gamma_{b}\right)$, and the total IIEE yield $\left(\gamma_{T}\right)$ obtained from Eqs. (1) and (2) are shown in Fig. 1. Measured yields as a function of the ion's atomic number reveal an oscillatory behavior with pronounced maxima and minima. In addition to the small hump near atomic number 11 , there are maxima of the yield at $Z_{1}$ equal to 19,27 , and 44, while minima occur near $Z_{1}$ equal to $13,22,29$, and 46 . We have also measured the yield from $\mathrm{F}^{2+}$ and $\mathrm{Cl}^{2+}$ as a check of any incident charge state dependence of the yield. No significant difference was observed. However, the $\bar{q}_{f}=2$ data are slightly smaller than the $\mathrm{F}^{3+}$ and $\mathrm{Cl}^{3+}$ data. Nevertheless, the data fall within the experimental uncertainties of the yield.

In Sternglass's theory [3], the ion-induced electron emission yield is proportional to the electronic stopping power $(d E / d X)_{e}$ and the proportionality constant is known as the material parameter

$$
\Lambda=\frac{\gamma}{\left(\frac{d E}{d x}\right)_{e}}
$$

The material parameter obtained from the ratio of the present measured yield and the tabulated electronic stopping powers [25] as a function of the projectile atomic number $\left(Z_{1}\right)$ is shown in Fig. 2. Contrary to the predictions of existing theories $[3,4], \Lambda$ is not constant, but rather oscillates as a function of $Z_{1}$. Indeed, the $Z_{1}$ oscillation observed is not correlated with oscillations in the electronic stopping power. Present results are also in disagreement with those of Clouvas et al. [26,27], who concluded recently that for 


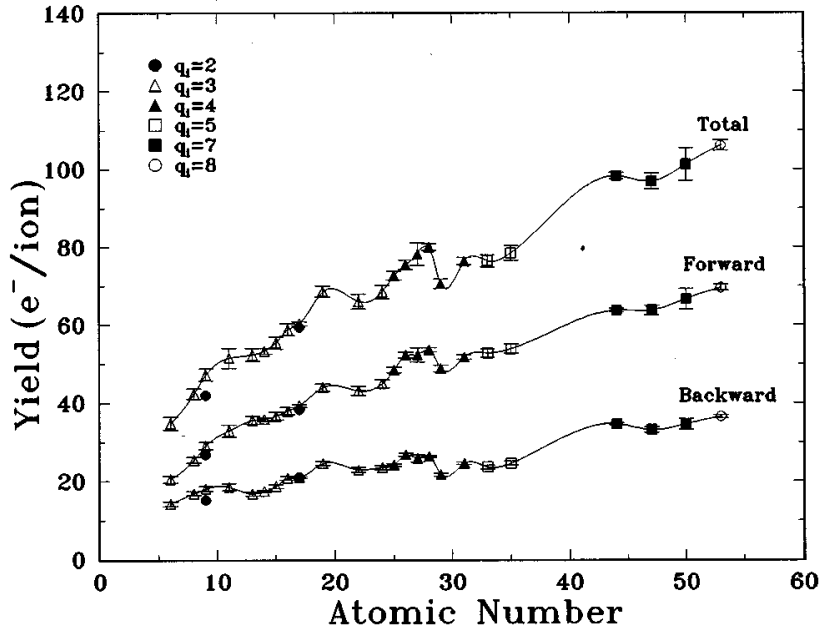

FIG. 1. Forward $\left(\gamma_{f}\right)$, backward $\left(\gamma_{b}\right)$, and total $\left(\gamma_{T}\right)$ ioninduced electron emission yields for ions of identical velocity $\left(v=2 v_{0}\right.$, where $v_{0}$ is the Bohr velocity) incident on sputtercleaned $50 \mu \mathrm{g} / \mathrm{cm}^{2}$ carbon foils as a function of the ion's atomic number $\left(Z_{1}\right)$. Data from $\mathrm{Ti}^{3+}, \mathrm{Fe}^{4+}, \mathrm{Ni}^{4+}, \mathrm{Ru}^{7+}, \mathrm{Ag}^{7+}, \mathrm{Sn}^{7+}$ were taken at a pressure of $1 \times 10^{-9}$ Torr. The other data were taken at pressures ranging from $8 \times 10^{-9}$ to $2 \times 10^{-8}$ Torr. Incident charge states are identified in the figure by different symbols, while the final charge states are distributed with a mean value given in Ref. [36]. ${ }_{17} \mathrm{Cl}$ and ${ }_{9} \mathrm{~F}$ were measured with two different incident charge states $\left(q_{i}\right)$ to verify the insensitivity of the yield to the incident charge state. The solid curve is drawn to guide the eye.

$1 \leqslant Z_{1} \leqslant 35$ at a high vacuum $\left(10^{-6}\right.$ Torr $)$ the material parameter obtained using the total yield $\left(\gamma_{T}\right)$ saturates for $Z_{1} \geqslant 6$. According to the data of this work shown in Fig. 2, the material parameter oscillates for $Z_{1}$ values well above 6 . Fastrup, Hvelplund, and Sautter [28] had earlier measured the stopping cross section in carbon of $6 \leqslant Z_{1} \leqslant 20$ ions of constant velocities $\left(v=0.41 v_{0}, 0.63 v_{0}, 0.91 v_{0}\right.$, and $\left.1.1 v_{0}\right)$. Even though their projectile velocities were much smaller than those of the present work, the measured elec-

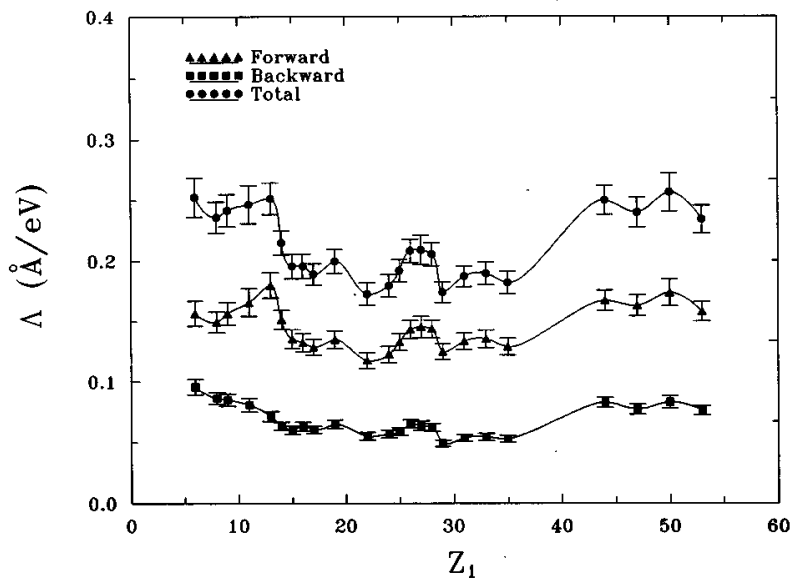

FIG. 2. Ratio of ion-induced electron emission yields $(\gamma)$ to electronic stopping powers $\left(S_{e}\right): \Lambda_{f}=\gamma_{f} / S_{e}, \Lambda_{b}=\gamma_{b} / S_{e}$, and $\Lambda_{t}=\gamma_{t} / S_{e}$ as a function of $Z_{1} . S_{e}$ is the tabulated stopping power [25]. The solid curve is drawn to guide the eye. tronic stopping cross sections of Fastrup, Hvelplund, and Sautter as a function of $Z_{1}$ show oscillatory features that are not present in the tabulated bulk stopping power cross sections [25]. One possible explanation for the oscillations in $\Lambda$ might lie in the lack of accurate tabulated bulk stopping powers for computing $\Lambda$. It may be that the tabulated bulk stopping powers do not accurately reveal all the projectiledependent features, such as screening and charge exchange. Simultaneous measurement of the yield and the electronic stopping powers might shed some light on this phenomenon.

A projectile nuclear charge dependence of the material parameter $\left(\Lambda_{f, b}\right)$ was noticed earlier by Rothard et al. $[18,29]$. They suggested the need to define a true material parameter independent of $Z_{1}$. In the context of the transport theory $[4,13]$, they have introduced, empirically, $Z_{1}$-dependent factors $C_{f}\left(Z_{1}\right), C_{b}\left(Z_{1}\right)$, and $C_{T}\left(Z_{1}\right)$ into the yield equations; thus,

$$
\begin{gathered}
\gamma_{f}=\Lambda\left(Z_{2}\right)(1-\beta) C_{f}\left(Z_{1}\right)\left(\frac{d E}{d x}\right)_{e}, \\
\gamma_{b}=\Lambda\left(Z_{2}\right) \beta C_{b}\left(Z_{1}\right)\left(\frac{d E}{d x}\right)_{e} .
\end{gathered}
$$

At sufficiently high projectile velocity, $\beta$ does not depend on $Z_{1}$. In terms of the material parameter for protons of the same velocity as the ions, the projectile dependent factors are given by

$$
C_{b}=\frac{\Lambda_{b}\left(Z_{1}\right)}{\Lambda_{b}\left(Z_{1}=1\right)}, \quad C_{f}=\frac{\Lambda_{f}\left(Z_{1}\right)}{\Lambda_{f}\left(Z_{1}=1\right)}, \quad C_{T}=\frac{\Lambda_{t}\left(Z_{1}\right)}{\Lambda_{t}\left(Z_{1}=1\right)} .
$$

According to this model, the parameter $C$ describes deviations of the near surface stopping powers from the tabulated bulk values and is claimed to account for several mechanisms such as charge exchange, screening effects, projectile ionization, and even molecular orbital effects.

The parameter $C_{T}$ as a function of $Z_{1}$ obtained from the present work is shown in Fig. 3. The proton material parameter used to compute $C_{T}$ was obtained from Meckbach's yield data [30]. Also shown are data from other authors $[26,27,29]$. The data from these authors show a decrease of $C_{T}$ with $Z_{1}$. However, the data from Clouvas et al., obtained at a modest vacuum ( $10^{-6}$ Torr), reach an equilibrium value $C_{T}\left(Z_{1} \geqslant 6\right)=0.57$, in contrast with the data from Rothard et al., obtained at an ultrahigh vacuum $\left(10^{-9}\right.$ Torr), which converge to $C_{T}\left(Z_{1}>6\right) \approx 0.4$. Rothard attributes the difference in the absolute value of $C_{T}$ to the strong dependence of the electron emission on the surface contamination and structure. Our data, obtained at pressures at or below $10^{-9}$ Torr, show $C_{T}$ not to be a decreasing function of $Z_{1}$ but rather to have a nonmonotonic dependence on $Z_{1}$. The data from Clouvas et al. seem to be close to our average in the interval $6 \leqslant Z_{1}<35$. For $Z_{1}>35$, while still oscillating, our data show an increase of $C_{T}$ with $Z_{1}$. According to Eqs. (1) and (2), $C_{T}$ is a function of $Z_{1}$ alone, and-contrary to Rothard's assessments-no vacuum condition effect that should manifest itself on the surface of the target is expected to influence the magnitude of $C_{T}$. 


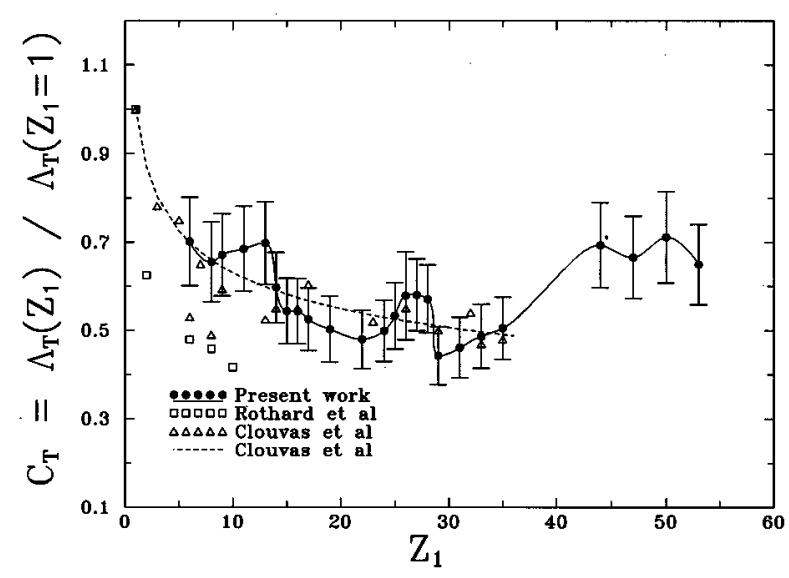

FIG. 3. The parameter $C_{T}\left(Z_{1}\right)=\Lambda_{T}\left(Z_{1}\right) / \Lambda_{T}\left(Z_{1}=1\right)$ plotted as a function of $Z_{1} . \Lambda\left(Z_{1}=1\right)$ was obtained from the result of Meckbach [30]. The solid curve is drawn to guide the eye. The dashed line is $C=Z_{1}^{-0.2}$ given in Ref. [27].

Experimentally, the ion-induced electron emission yield computed using Eqs. (1) and (2) is proportional to the equilibrium mean charge state $\left(\overline{q_{f}}\right)$ of the ion. Therefore, an accurate knowledge of $\overline{q_{f}}$ is essential to computation of a correct yield. Prior to the present work, instead of measuring $\overline{q_{f}}$, most of the previously reported yields $[15,26,27]$ relied heavily on a monotonic empirical formula $[31,32]$ for $\overline{q_{f}}$. However, for ions of identical velocity $\left(v=v_{0}\right)$ incident on carbon foil, Lennard and Phillips [33] have reported that $\overline{q_{f}}$ increases strongly with $Z_{1}$ with a broad peak at $Z_{1} \sim 15$. Similarly, Shima et al. $[32,34,35]$ reported oscillations of $\bar{q}_{f}$ as a function of $Z_{1}$ for ions of $0.55,1$, and $2 \mathrm{MeV} / \mathrm{u}$ with oscillation maxima occurring for the closed-shell ions. In the light of these findings about $\overline{q_{f}}$, yields for ions exhibiting $\overline{q_{f}}$ oscillations are expected to be in error if $\bar{q}_{f}$ is obtained by smooth extrapolation. The authors of this work have investigated this effect in a previous report [36]. The results of their study are reported below.

The measured mean charge state as a function of $Z_{1}$ exhibits an oscillatory behavior with a strong peak in the region $24 \leqslant Z_{1} \leqslant 29$ [36]. Indeed, this is the same $Z_{1}$ interval where we observed the strong peak in the yield. Our projectile velocity was lower than the range in which shell effects [37] reportedly can play a role in the oscillations observed in $\overline{q_{f}}$. However it should not be surprising to observe oscillations in the mean charge state as a function of the atomic number, since such oscillations have been observed at much lower velocities and much higher velocities as well. The exact origin of the oscillations is still controversial and unclear, but the present data would suggest that the mean charge state of the ion after the foil is not representative of the ion's charge state in the foil. Post-foil-Auger processes are shown [36] to be a more reasonable cause of some peaks observed in the yield measurements. The significance of the nonmonotonic dependence of $\overline{q_{f}}$ should be emphasized, since it is the source of a significant systematic experimental error in most of the previously published data. In the work reported here, the IIEE yield and the mean charge state are measured simultaneously, avoiding altogether the aforementioned systematic error.
The implications of the results of this work for isobar detection are significant. In order to distinguish between isobars differing in atomic number by $\Delta Z_{1}$ using IIEE, the standard error in the measured atomic number $\delta Z_{1}$ must be significantly smaller than this $\Delta Z_{1}$. The quantity $\delta Z_{1}$ is related to the standard error in the yield by

$$
\delta Z_{1}=\frac{\delta \gamma}{\left(\frac{d \gamma}{d Z_{1}}\right)}
$$

It is clear upon reflection that the highest resolution (minimum $\delta Z_{1}$ ) will occur where $d \gamma / d Z_{1}$ is largest and $\delta \gamma$ smallest. Therefore, at the maxima of the $\gamma$ vs $Z_{1}$ curve, where $d \gamma / d Z_{1}=0$, little—if any-discrimination will be possible. On average, $\delta \gamma$ can be as low as $1 \%$ of $\gamma$. With $\left(d \gamma / d Z_{1}\right) \geqslant 2\left(e^{-} /\right.$ion $) /$atomic number, a resolution of better than $0.5 \%$ for $\delta Z_{1} / Z_{1}$ will be expected. Such regions exist just below the local maxima of the curve. On the other hand, the expected fluctuation in the electron yield for single events is anticipated to be much larger than the error in the mean. This fact may limit the utility of the proposed discrimination procedure. The subject of the statistics of the process promises to be a fruitful area for further investigation. The issue of the correlation of energy loss, electron yield, and equilibrium charge state is also a potential topic for future work.

\section{CONCLUSION}

The $Z_{1}$ dependence, that is, the dependence on the incident ion's nuclear charge, of ion-induced electron emission yields has been studied for constant velocity ions incident on $50 \mu \mathrm{g} / \mathrm{cm}^{2}$ carbon foils. Measured yields as a function of $Z_{1}$ reveal oscillatory features, some of which are correlated with similar oscillations in the mean charge state $\left(\bar{q}_{f}\right)$. These oscillations are also evident in the material parameter $\Lambda$, the yield divided by the target electronic stopping power.

As a consequence of these findings, one must conclude that the previous literature that relied on semiempirical $\overline{q_{f}}$ data may be potentially in error. The absence of peaks in the previous data may be a masking artifact of the use of the tabulated values which relied on the extrapolated data. By the simultaneous measurement of $\overline{q_{f}}$ and the electron currents one is able to make accurate measurements of $\gamma$, however.

\section{ACKNOWLEDGMENTS}

The authors would like to thank Dr. Jagdish Prasad, Dr. R. B. Escue, Dwight Maxson, and Jianyue Jin for their technical assistance. The present work is supported in part by the National Science Foundation Grants No. DMR-8812331, INT-8917946, and ECD-9003099, the Office of Naval Research Grants No. N00014-89-J-1309, N00014-89-J-1344, N00014-90-1691, and N00014-91-J-1785, Texas Instruments Incorporated, Texas Utilities Electric Inc., International Digital Modeling Corp., North Texas Research Institute, and the Robert A. Welch Foundation. 
[1] J. S. Allen, Phys. Rev. 55, 336 (1939).

[2] J. Schader, B. Kolb, K. D. Sevier, and K. O. Groeneveld, Nucl. Instrum. Methods 151, 563 (1978).

[3] E. J. Sternglass, Phys. Rev. 108, 1 (1957).

[4] J. Schou, Phys. Rev. B 22, 2141 (1980).

[5] H.-G. Clerc, H. J. Gehrhardt, L. Richter, and K. H. Schmidt, Nucl. Instrum. Methods 113, 325 (1973).

[6] S. Matteson, D. K. Marble, L. S. Hodges, N. Y. Hajsaleh, A. M. Arrale, M. R. McNeir, J. L. Duggan, F. D. McDaniel, and J. M. Anthony, Nucl. Instrum. Methods B 45, 575 (1990).

[7] C. E. Kuyat, in Methods of Experimental Physics, edited by B. Bederson and W. L. Fite (Academic, New York, 1968), Vol. 7A, p. 575.

[8] S. Matteson and M. A. Nicolet, Nucl. Instrum. Methods 160, 301 (1979).

[9] R. A. Baragiola, E. V. Alonso, O. Auciello, J. Ferron, G. Lantschner, and A. Oliva-Florio, Phys. Lett. 67A, 211 (1978).

[10] E. Veje, Nucl. Instrum. Methods 194, 433 (1982).

[11] R. A. Baragiola, E. V. Alonso, J. Ferron, and A. Oliva-Florio, Surf. Sci. 90, 240 (1979).

[12] H. D. Hagstrum, Phys. Rev. 96, 336 (1954).

[13] J. Schou, Scanning Electron Micros. 2, 607 (1988).

[14] P. Sigmund and S. Tougaard, Inelastic Particle-Surface Collisions, edited by E. Taglauer and W. Heiland (Springer, Heidelberg, 1981), p. 2.

[15] A. Clouvas, H. Rothard, M. Burkhard, K. Kroneberger, C. Biedermann, J. Kemmler, K. O. Groeneveld, R. Kirsch, P. Misaelides, and A. Katsanos, Phys. Rev. B 39, 6316 (1989).

[16] D. Hasselkamp, K. G. Lang, A. Scharmann, and N. Stiller, Comments At. Mol. Phys. 21, 241 (1988).

[17] J. E. Borovsky, D. J. McComas, and B. L. Baraclough, Nucl. Instrum. Methods B 30, 191 (1988).

[18] H. Rothard, K. Kroneberger, A. Cluovas, E. Veje, P. Lorenzen, N. Keller, J. Kemmler, W. Meckbach, and K. O. Groeneveld, Phys. Rev. A 41, 2521 (1990).

[19] P. Koschar, K. Kroneberger, A. Clouvas, M. Burkhard, W. Meckbach, O. Heil, J. Kemmler, H. Rothard, K. O. Groen- eveld, R. Schramm, and H.-D. Betz, Phys. Rev. A 40, 3632 (1989).

[20] C. R. Shi, H. S. Toh, D. Lo, R. P. Livi, M. H. Mendenhall, D. Z. Zang, and T. A. Tombrello, Nucl. Instrum. Methods B 9, 263 (1985).

[21] U. Fehn, Int. J. Mass Spectrom. Ion Phys. 21, 1 (1976).

[22] M. M. Ferguson and W. O. Hofer, Radiat. Eff. Defects Solids 109, 273 (1989).

[23] W. O. Hofer, Scanning Micros. Suppl. 4, 265 (1990).

[24] A. M. Arrale, Z. Y. Zhao, J. F. Kirchhoff, D. K. Marble, D. L. Weathers, F. D. McDaniel, and S. Matteson, Nucl. Instrum. Methods Phys. B 89, 437 (1994).

[25] J. F. Ziegler, J. P. Biersack, and U. Littmark, The Stopping Ranges of Ions in Matter (Pergamon, New York, 1985).

[26] A. Clouvas, A. Katsanos, B. Farizon-Mazuy, M. Farizon, and M. J. Gaillard, Phys. Rev. B 43, 2496 (1991).

[27] A. Clouvas, A. Katsanos, B. Farizon-Mazuy, M. Farizon, M. J. Gaillard, and S. Ouaskit, Phys. Rev. B 48, 6832 (1993).

[28] B. Fastrup, P. Hvelplund, and C. Sautter, Mat. Fys. Medd. Dan. Vid. Selsk. 35, 10 (1966).

[29] H. Rothard, J. Schou, and K. O. Groeneveld, Phys. Rev. A 45, 1701 (1992).

[30] W. Meckbach, J. Phys. B 8, L344 (1975).

[31] K. Shima, T. Shikata, and T. Mikuma, Nucl. Instrum. Methods 200, 605 (1982).

[32] K. Shima, N. Kuno, and M. Yamanouchi, Phys. Rev. A 40, 3557 (1989).

[33] W. N. Lennard and D. Phillips, Phys. Rev. Lett. 45, 176 (1980).

[34] K. Shima, N. Kuno, T. Kakita, and M. Yamanouchi, Phys. Rev. A 39, 4316 (1989).

[35] K. Shima, N. Kuno, M. Yamanouchi, and H. Tawara, At. Data Nucl. Data Tables 51, 173 (1992).

[36] A. M. Arrale, Jianyue Jin, Z. Y. Zhao, D. L. Weathers, F. D. McDaniel, and S. Matteson, Phys. Rev. A 51, 3355 (1995).

[37] C. M. Moak, H. O. Lutz, L. B. Bridwell, L. C. Northcliffe, and S. Datz, Phys. Rev. Lett. 45, 176 (1980). 